Rev. Pat. Trop.24 ( 2 ): 275 - 281, jul/dez. 1995

\title{
EFETIVIDADE DE BACTIVEC, A BASE DE Bacillus thuringiensis H-14 NO CONTROLE DE Simulium pertinax (Diptera, Simuliidae)
}

\author{
Carlos Fernando S. Andrade ${ }^{\prime} \&$ Jairo Campos $G^{2}$.
}

\section{RESUMO}

Bactivec ${ }^{\circledR}$ (Biolarvicida BT H-14, Labiofam) tem sido avaliado e usado contra diversas espécies de culicídeos em Cuba à razão de $10 \mathrm{ml}$ de produto por $\mathrm{m}^{2}$. No presente estudo o produto foi avaliado contra larvas de Simulium pertinax em um sistema de calhas com vazão média de $32 \mathrm{l} / \mathrm{min}$, no próprio riacho, em concentrações entre 36 e 288 ppm/10min. Os produtos Teknar® (BT H-14, Zeneca Saúde Pública) e Abate 500-E® (Temephos, American Cyanamid) foram usados como referência nas concentrações de $1,2 \mathrm{ppm} / 10 \mathrm{~min}$ e $0,1 \mathrm{ppm}$ i.a./10 min respectivamente. As concentrações de Bactivec causaram entre $37,8 \%$ e $90,5 \%$ de mortalidade após 24 horas, enquanto que Teknar causou $92,5 \%$ e Abate $3,1 \%$. Confirma-se no presente estudo a resistência dessa população de $S$. pertinax ao organofosforado e a efetividade dos produtos a base de BT H-14. Considerando-se o tamanho médio dos riachos aonde se cria $S$. pertinax, torna-se necessário preparados mais concentrados de Bactivec $®$ para viabilizar seu uso em programas de controle no Brasil.

UNITERMOS: Borrachudo, controle microbiano.

\section{INTRODUÇÃO}

Após sua primeira descoberta do solo em Israel em 1976 (GOLDBERG \& MARGALIT, 1977), diversos isolados de Bacillus thuringiensis var. israelensis (BTI) (sorotipo H-14) foram feitos em todo o mundo, incluindo de larvas de quironomídeos no Brasil (ANDRADE \& HABIB, 1985) ou do solo no Nepal a $6.000 \mathrm{~m}$ de altitude (MORRILL JR., 1990). Graças à elevada toxicidade desse sorotipo às larvas de dípteros aquáticos, e ao sucesso na industrialização de outras

\footnotetext{
${ }_{2}^{1}$ Dept $^{\circ}$ de Zoologia, Inst ${ }^{\circ}$. de Biologia, UNICAMP. Cx.P. 6019 Campinas/SP

${ }^{2}$ Pós-Graduação em Biologia Celular, IB-UNICAMP. Campinas/SP
} 
variedades para o controle de lepidópteros praga, poucos anos após a sua descoberta já existiam produtos comerciais a base de BTI.

O surgimento de resistência aos organofosforados nas larvas de simulídeos vetores de oncocercose na África, no final da década de 70, deu enorme impulso ao desenvolvimento de formulações de BTI para o controle de borrachudos (JAMNBACK, 1981). A deteç̧ão de resistência no Brasil das larvas de Simulium pertinax ao organofosforado Abate 500E (RUAS NETO, 1984 e ANDRADE, 1989a), fez com que os principais programas de manejo, públicos ou privados, passassem também a depender do BTI (ANDRADE, 1989b).

O mercado mundial de produtos a base de BTI tem sido amplamente dominado por três fabricantes. Os laboratórios Abbott produzem diversas formulações de Vectobac ${ }^{\circledR}$ e comercializam no Brasil uma suspensão aquosa com 1.200 Unidades Internacionais de Potência por miligrama (UIP/mg). A Zoecon Corporation distribui o produto Teknar ${ }^{\circledR}$, também suspensão aquosa com 3.000 Unidades Aedes Aegypti (UAA) por miligrama (=1.200 UIP/mg), comercializada no Brasil pela Zeneca Saúde Pública, e ainda a Novo Nordisk, fora do Brasil, comercializa o Skeetal® e o Bactimos®.

Afora esses produtos internacionais, vários outros países como Itália, Israel, antiga União Soviética, China e Cuba também produzem preparados industriais. Esforços para se produzir o BTI no nosso país tem sido feitos em instituições como Fiocruz (Rio de Janeiro/RJ), Cenargen (Brasília/DF), Emcapa (Vitória/ES), Unesp (Jabotibacal/SP) e Unicamp-CPQBA (Campinas/SP). No entanto por terem produção limitada e/ou problemas com a formulação e estabilidade dos preparados, não têm participado de programas de controle de borrachudos.

O laboratório cubano Labiofam desenvolve em conjunto com o Instituto de Medicina Tropical "Pedro Koury" (Havana, Cuba) desde 1978, o produto a base de BTI "Biolarvicida BTH 14" ou Bactivec, com titulação de pelo menos $10^{8}$ esporos $/ \mathrm{ml}$. Tal produto tem sido usado contra diferentes culicídeos em Cuba à razão de $10 \mathrm{ml} / \mathrm{m}^{2}$ com excelentes resultados (G. MONTERO L., comunicação pessoal) mas não tem sido avaliado contra simulídeos. O presente trabalho trata da avaliação de efetividade de Bactivec comparativamente com Teknar e Abate 500E, no controle de larvas de Simulium pertinax, sob condições de campo.

\section{MATERIAIS E MÉTODOS}

O ensaio foi realizado no riacho Cachoeirinha tributário do rio Jaguari (Serra das Cabras) no Município de Morungaba/SP. Foi montado no leito do riacho um sistema de calhas paralelas de cimento amianto de $1,2 \mathrm{~m}$ de comprimento cada, de fundo arredondado e largura de $15 \mathrm{~cm}$. A entrada de água nas calhas foi manejada de forma a ficarem todas com vazão de cerca de $32 \mathrm{1} / \mathrm{min}$, em uma seç̧ão de 11,5
ANDRADE, C.F.S.; CAMPOS G, J. Efetividade de BACTIVEC, a base de Bacillus thuringiensis $\mathrm{H}-14$ no controle de Simulium pertinax (Diptera, Simuliidae). Rev. Pat. Trop.24 (2 ): $275-281$, jul/dez. 1995

$\mathrm{cm}^{2}$. As vazões foram calculadas coletando-se com um balde graduado a água despejada por cada rampa. As larvas de $S$. pertinax foram coletadas nas cercanias desse sistema e transferidas para as calhas, (cerca de 100 larvas/calha) evitando-se colonizar o quarto superior destas. As aplicações foram feitas duas horas após o término da transferência das larvas para as rampas, para permitir que se aclimatassem. Para as avaliações foram descartadas as larvas menores, nos primeiros estádios e usadas duas rampas para cada tratamento e como controle.

Os produtos avaliados, suas características e concentrações aplicadas nas rampas encontram-se na Tabela 1.

TABELA 1. Produtos comerciais, ingredientes ativos e concentrações avaliadas em aplicações de 10 minutos de duração contra larvas de Simulium pertinax em sistema de rampas no leito do riacho.

\begin{tabular}{ccc}
\hline PRODUTO & INGREDIENTE ATIVO & CONCENTRAÇÃO / TEMPO \\
\hline BACTIVEC & BTI & $36,72,144$ e $288 \mathrm{ppm} / 10 \mathrm{~min}$. \\
TEKNAR & BTI & $1,2 \mathrm{ppm} / 10 \mathrm{~min}$. \\
ABATE 500E & TEMEPHOS & $0,1 \mathrm{ppm}$ i.a. $/ 10 \mathrm{~min}$. \\
\hline
\end{tabular}

As concentrações do produto Bactivec foram estabelecidas levando-se em consideração a recomendação do fabricante de se usar contra culicídeos $100 \mathrm{l} / \mathrm{ha}$, enquanto que para os produtos com $1.200 \mathrm{UIP} / \mathrm{mg}$, como Teknar e Vectobac é recomendado entre 0,5 a 2,0 ou $2,5 \mathrm{l} / \mathrm{ha}$, representando portanto entre 40 a 200 vezes menos produto.

As avaliações de mortalidade e desprendimento foram feitas 4 e 24 horas após a aplicação.

\section{RESULTADOS E DISCUSSÃO}

As recomendações de uso dos produtos Teknar e Vectobac para o controle de simulídeos são em geral vagas, pois deve-se considerar vários fatores intrínsecos, principalmente quanto às características fisicas e biológicas do curso d'água. Assim, em rios de águas limpas, mais caudalosos e sem obstáculos como tanques ou vegetação, pode-se usar concentrações menores com um bom carreamento do produto. Ao contrário, em riachos menores e acidentados, com águas turvas ou muita vegetação marginal ou mesmo aquática, recomenda-se maiores concentrações. Assim, encontra-se recomendado nos folhetos técnicos concentrações entre 0,5 e 25 ppm. Quanto ao tempo de exposição, os catálogos recomendam entre 1 a 20 minutos de tratamento. 
O organofosforado Temephos tem sido recomendado para o controle de simulídeos em concentrações entre 0,03 e 0,1 ppm i.a./10min (Cyanamid, 1980).

No presente trabalho pode-se observar já 4 horas após a aplicação, que tanto o produto Teknar como a maior concentração de Bactivec causaram mortalidades elevadas, enquanto que nenhuma mortalidade ocorreu devida ao tratamento com o organofosforado Abate. As concentrações inferiores de Bactivec causaram entre 0,0 e $35,0 \%$ de mortalidade. A porcentagem de desprendimento foi semelhante em todas as rampas tratadas com BTI e pouco significativa nas rampas tratadas com Abate $500 \mathrm{E}$ ou na testemunha (Tabela 2.).

Vinte e quatro horas após a aplicação a eficiência do tratamento pelo Teknar e pela maior concentração de Bactivec ficou pouco aumentada, não atingindo $100 \%$. Abate continuou com reduzida eficiência e as concentrações intermediárias de Bactivec tiveram eficiências entre cerca de 37 a $85 \%$. As porcentagens de desprendimento ficaram em geral aumentadas, variando entre cerca de 12 a $30 \%$ para os tratamentos a base de BTI e em cerca de 3,0\% para o Abate 500E. Não houve mortalidade e nem desprendimento nas rampas testemunhas 24 horas após as aplicações ( Tabela 2)

TABELA 2. Porcentagens de desprendimento (DESP.) e de mortalidade (MORT.) em larvas de Simulium pertinax 4 e 24 horas após o tratamento com o organofosforado Abate e com produtos a base de Bacillus thuringiensis $\mathrm{H}-14$ (BTI).

\begin{tabular}{cccccccc}
\hline & & \multicolumn{2}{c}{ 4 HORAS APÓS } & & \multicolumn{2}{c}{ 24 HORAS APÓS } \\
PRODUTO & Conc./10 min & \% DESP. & \% MORT. & & \% DESP. & \% MORT. \\
\hline ABATE 500E & $0,1 \mathrm{ppm}$ i.a. & 0,7 & 0,0 & & 3,1 & 0,5 \\
TEKNAR & $1,2 \mathrm{ppm}$ & 10,8 & 89,1 & & 25,0 & 92,4 \\
BACTIVEC & $36 \mathrm{ppm}$ & 8,2 & 0,0 & & 29,8 & 37,8 \\
BACTIVEC & $72 \mathrm{ppm}$ & 8,0 & 22,0 & & 12,3 & 70,5 \\
BACTIVEC & $144 \mathrm{ppm}$ & 13,1 & 35,0 & & 23,8 & 85,7 \\
BECTIVEC & $288 \mathrm{ppm}$ & 8,5 & 88,0 & & 15,2 & 90,5 \\
TESTEMUNHA & - & 1,7 & 0,0 & & 0,0 & 0,0 \\
\hline
\end{tabular}

A concentração de Abate empregada correspondia à maior recomendada pelo fabricante. Estudos preliminares (CAMPOS et al., 1995) já haviam indicado que nessa localidade $S$. pertinax não apresentava uma completa susceptibilidade ao organofosforado. O curso d'água situa-se em área rural, com atividade primária de pecuária e não tem sido sujeito a tratamento larvicida, apesar do ataque antropofílico de borrachudos ser bastante incômodo na região. Frequentemente moradores da fazenda necessitam de cuidados médicos devido à quantidade de picadas ou às infecções secundárias surgidas em decorrência destas. A explicação mais plausível
ANDRADE, C.F.S.; CAMPOS G, J. Efetividade de BACTIVEC, a base de Bacillus thuringiensis H-14 no controle de Simulium pertinax (Diptera, Simuliidae). Rev. Pat. Trop.24 ( 2 ): 275 - 281, jul/dez. 1995

para a resistência detectada nessa região seria devido ao carreamento de resíduos de produtos agrícolas para o riacho, promovendo assim uma resistência cruzada. Esse mesmo fenômeno já foi verificado para outras situações (KURTAK et al., 1987, LIES, 1988 e ANDRADE, 1989a).

A concentração de Teknar estabelecida no presente estudo $(1,2 \mathrm{ppm} / 10 \mathrm{~min})$ foi intencionalmente baixa considerando-se a vazão de $32 \mathrm{l} / \mathrm{min}$ das rampas, para permitir maior sensibilidade e comparação com as de Bactivec. Segundo o fabricante, para cursos com pequena vazão deveriam ser usadas as maiores concentrações ( $25 \mathrm{ppm})$. No programa estadual de controle de simulídeos do Rio Grande do Sul, o uso de BTI sequer é recomendado em cursos com vazões entre 10 a $160 \mathrm{l} / \mathrm{min}$. Entre 170 e $312 \mathrm{l} / \mathrm{min}$, a recomendação de uso é de $50 \mathrm{ppm} / 1 \mathrm{~min}$ (RUAS NETO, 1991), que poderia ser também assumida como $5 \mathrm{ppm} / 10 \mathrm{~min}$. Dessa forma, não era realmente de se esperar que a eficiência de Teknar fosse total.

Embora tenha sido assumido para avaliações de campo que a relação de larvas desprendidas após a aplicação seja baixa (ANDRADE, 1989a), o presente estudo indica que ao menos para o sistema de rampas esses valores podem ser consideráveis, variando entre cerca de $8 \%$ após 4 horas e $25 \%$ após 24 horas. De qualquer forma, considerando-se que não houve desprendimento de larvas nas rampas testemunhas e que a paralisia causada pela intoxicação por BTI seja irreversível, deve-se considerar para todos os efeitos que esse desprendimento resulta em controle.

Enquanto o produto Vectobac (Abbott) apresenta 1,2\% de ingrediente ativo (BTI) e Teknar (Zoecon/Zeneca) tem 1,6\% de i.a., o produto cubano Bactivec é formulado com $0,5 \%$ de i.a. Tais quantidades de BTI no formulado final não representam de qualquer modo a potência dos preparados. Ensaios padrão com larvas de Aedes aegypti e comparativos ao BTI 'standard' IPS-82 (Instituto Pasteur) é que irão definir as potências em termos das Unidades Internacionais (UIP) (MCLAUGHLIN et al., 1984 e DULMAGE et al., 1990). De qualquer forma, o paralelo estabelecido em função das recomendações do produto cubano para culicídeos, mostrou que Bactivec é efetivo contra larvas de simulídeos. A maior concentração avaliada desse produto $(288 \mathrm{ppm})$ teve eficiência comparável à de Teknar na concentração de $1,2 \mathrm{ppm}$. A grosso modo, pode-se esperar que em sistemas maiores essa relação de cerca de 250 vezes entre os dois produtos também ocorra. Nesse caso, se considerarmos um riacho hipotético de tamanho médio, típico criadouro de larvas de $S$. pertinax, com cerca de $1 \mathrm{~m}$ de largura e $10 \mathrm{~cm}$ de profundidade média, e com velocidade de $1 \mathrm{~m} / \mathrm{s}$, a quantidade de Bactivec necessária para uma aplicação seria de 18 litros; impraticável para as condições operacionais de campo. Além da concentração de ingrediente ativo nos produtos a base de BTI, os outros componentes, inertes, corroboram com a eficiência do preparado, como sua capacidade de carreamento (transporte). As formulações mais adequadas seriam 
ANDRADE, C.F. S ; CAMPOS G, J. Efetividade de BACTIVEC, a base de Bacillus thuringiensis H-1 4 no controle de Simulium pertinax (Diptera, Simuliidae). Rev. Pat. Trop.24 (2 ): 275 - 281, jul/dez. 1995

aquelas mais leves, capazes de serem carregadas pela água por distâncias maiores abaixo do ponto de aplicação. Assim, pode-se concluir que novas formulações de Bactivec, mais concentradas, seriam necessárias para os programas de controle de simulídeos. Tais formulações necessitariam ainda ser avaliadas quanto ao carreamento, para riachos criadouros de $S$. pertinax de diferentes características.

Seja em áreas de produção agrícola como as dos estados ao sul do Brasil, ou em áreas turísticas como o litoral de São Paulo e Rio de Janeiro, o controle de $S$. pertinax é imperativo, tanto do ponto de vista econômico, social como de atendimento primário à saúde da população. Assim, o desenvolvimento de novas opções para o controle biológico desse inseto é fundamental como ponto de partida para os programas de manejo.

\section{SUMMARY}

EFECTIVITY OF BACTIVEC, A Bacillus thuringiensis $\mathrm{H}-14$ based product in controlling Simulium pertinax (DIPTERA, SIMULIIDAE).

The product Bactivec $®$ (Biolarvicida BT $\mathrm{H}-14$, Labiofam) has been evaluated and used in Cuba against many culicid species at $10 \mathrm{ml} / \mathrm{m}^{2}$. In the present study Bactivec was evaluated against Simulium pertinax larvae in a system of troughs assembled in the stream bed (Morungaba/S.P., Brazil). The mean water flow in the troughs was $32 \mathrm{l} / \mathrm{min}$. Four Concentrations of Bactivec ranging from 36 to 288 $\mathrm{ppm} / 10 \mathrm{~min}$ were evaluated. The products Teknar (BT H-14, Zoecon Corp./ Zeneca Saúde Pública) and Abate 500-E (Temephos, American Cyanamid) were also evaluated respectively at $1.2 \mathrm{ppm} / 10 \mathrm{~min}$ and $0.1 \mathrm{ppm}$ a.i./10min as reference. The established concentrations of Bactivec caused mortalities ranging from $37.8 \%$ to $90.5 \%$ while Teknar caused $92.5 \%$ and Abate $500 \mathrm{E}$ caused $3.1 \%$. Resistance in such population of $S$. pertinax to the chemical and the efectivity of the BT H-14 products were confirmed. Considering the mean size of a typical stream where $S$. pertinax larvae breed it was concluded that highly concentrated preparations are needed in order to attend control programs in Brazil.

KEYWORDS: Blackfly, microbial control.

\section{REFERÊNCIAS BIBLIOGRÁFICAS}

ANDRADE, C.F.S. \& M.E.M. HABIB,. Primeiro registro de ocorrência do sorotipo $\mathrm{H}-14$ de Bacillus thuringiensis Berliner no Brasil. 12 Congr. Bras. Zool. (Campinas/SP). Resumos:125. 1985
ANDRADE, C.F S.; CAMPOS G, J. Efetividade de BACTIVEC, a base de Bacillus thuringiensis H-14 no controle de Simulium pertinax (Diptera, Simuliidae). Rev. Pat. Trop.24 (2 ): $275-281$, jul/dez. 1995

ANDRADE, C.F.S., Ecologia de Supressão de Populações de Culicídeos e Simulídeos. Tese de Doutoramento, Área de Ecologia, Instituto de Biologia, UNICAMP, 253-89, 1989a.

ANDRADE, C.F.S.,. Manejo Integrado de Borrachudos. Anais do 11 Congr. Bras. Entomol., Campinas, SP, 1987, 3: 141-157, 1989b

CAMPOS G., J., RECCO-PIMENTEL, S.M. \& ANDRADE, C.F.S., Polytene chomosome analysis of a population of Simulium pertinax (Diptera, Simuliidae). Revista Brasileira de Genética. (no prelo).CYANAMID, 1980. "Abate Larvicida". Cyanamid Agricultural Research Division. American Cyanamid Co., Manual Técnico, 39 p. 1995.

DULMAGE, T., YOUSTEN, A.A., SINGER, S. \& LACEY, L.A., Guidelines for production of Bacillus thuringiensis $\mathrm{H}-14$ and Bacillus sphaericus. UNDP/WHO/TDR/BCV/90.1. 58 p, 1990.

GOLDBERG, L.H. \& MARGALIT, J., A bacterial spore demonstrating rapid larvicidal activity against Anopheles sergentii, Uranotaenia unguiculata, Culex univittatus, Aedes aegypti and Culex pipiens. Mosquito Mews, 37:355-358. 1977.

JAMNBACK, H. The origins of blackfly control programmes. Em: " Blackflies The Future for Biological Methods in Integrated Control", M. Laird ed., Academic Press, N.Y. e Londres, 398 p, 1981.

KURTAK, D.; JAMNBACK, H.; MYER, R.; OCRAN, M. \& RENAUD, P., Evaluation of larvicides for the control of Simulium damnosum (Diptera: Simuliidae) in west Africa. J. Amer. Mosq. Control Assoc. 3:201-210, 1987.

LIES, J.D., Do agricultural insecticides select for insecticide resistance in mosquitoes? A look at the evidence. Parasitology Today, 4:s17-s20, 1988.

MCLAUGHLIN, R.E., DULMAGA, H.T., ALLS, R., COUCH, T.L., DAME, D.A., HALL, I.M., ROSE, R.I. \& VERSOI, P.L., U. S. standard bioassay for the potency assessment of Bacillus thuringiensis Serotype H-14 against mosquito larvae. Bull.of the ESA. (1984) : 26-29, 1984.

MORRILL Jr., A.W., News and Notes. J. Am. Mosq. Control Assoc. 6:154, 1990. 